\title{
VARIANTES Y MODULACIONES DE LAS SOLEDADES DE ANTONIO MACHADO
}

\author{
Dolores ROMERO LÓPEZ DE LAS HAZAS \\ Universidad de Salamanca
}

Como es bien sabido, la primera edición de las Soledades, realizada por la Imprenta de A. Álvarez en 1903, volvió a ser emitida con distintas solapas por la Imprenta Valero Díaz en 1904. Esta vez el ejemplar se vendía a una peseta, es decir , a mitad de precio que el original (Valle 1949, pág. 81); se trataba, pues, como apunta G. Ribbans, de una reventa del material en stock (Ribbans 1962, pág. 195). Desde su publicación, la crítica ha sentido gran interés por esta primicia literaria ${ }^{1}$. A pesar de ello, todavía no se ha hecho un estudio sistemático de las variaciones y modulaciones que presentan los poemas de 1903 que fueron seleccionados y publicados en la edición de 1907. No hay ningún poema original de la edición de 1903 que no haya cambiado, aunque sea mínimamente, en la edición de Pueyo. Por lo tanto, no se puede concluir, como en su día lo hizo Luis Cernuda, que "Machado nace formado enteramente y el paso del tiempo nada le añadirá, antes le quitará" (1957, pág. 111). Aunque es verdad que los mejores poemas de Antonio Machado fueron muy tempranos y en sus Soledades encontramos la mejor poesía del poeta, lo cierto es que, como apunta D. Dámaso Alonso, "en los primeros tiempos la releyó, corrigió y seleccionó con gran cuidado y muchas veces" (1975, pág. 607). En 1903-4? dice Antonio Machado en carta a Juan Ramón: "No estoy muy satisfecho de las cosas que hago últimamente. Estoy en un período de evolución y todavía no he

1. Juan Ramón Jiménez publica en El País de 1903 un artículo donde alaba la poesía del sevillano. Posteriormente se realizaron otros estudios entre los que hay que destacar el artículo de Lauxar (1929, pp. 225-42) y el que Dámaso Alonso publicó por pirmera vez en 1949, recopilado posteriormente en sus Obras completas de donde se cita en la presente investigación. En 1959 fue publicada la obra del Prof. Oreste Macrí Poesie di Antonio Machado; un año más tarde aparecía en Insula el estudio del Prof. Ricardo Gullón sobre "Las Soledades de Antonio Machado"; Geoffrey Ribbans publica “Antonio Machado's Soledades (1903); A critical Study" (1962) y en 1968, Rafael Ferreres publica la edición crítica de las Soledades. Hoy, gracias a la edición de Oreste Macrí podemos disponer de todas las variantes textuales de este primer libro de A. Machado. 
encontrado la forma de expresión de mi nueva poesía. Lo último que se domina es la forma" (Cit. por Gullón 1964, pág. 41).

Queda clara, pues, la intención del poeta por superarse formalmente durante la época de recomposición de su libro Soledades. Sin embargo, en la introducción a las Páginas escogidas decía Antonio Machado que era su costumbre "no volver nunca sobre lo hecho y no leer nada de cuanto escribo" (1940, pág. 29). Esta afirmación no puede aplicarse a su primer libro de poesías dado que en él realizó un gran número de correcciones.

En mi estudio parto de las variantes textuales que aparecen al contrastar las concordancias de las dos primeras ediciones de las Soledades. Teniendo en cuenta que "el estudio de las variantes de la obra de un autor puede arrojar luz sobre los procedimientos de la crítica textual" (Segre 1990, pp. 55-56), la intención básica de esta investigación es ver cómo a través de dos versiones distintas de una obra se puede demostrar la evolución sufrida por el autor y por ende del período literario al que este se empezó adscribiendo. La comparación de estas dos ediciones constata científicamene la depuración del Modernismo hacia una estética más personalizada y objetiva y además demuestra un mayor grado de conciencia crítica en el poeta de 1907 frente al joven escritor de 1903.

Por supuesto que soy de la opinión de Cesare Segre al afirmar que "no tiene sentido buscar en la confrontación entre dos variantes correspondientes los motivos del cambio, si no se tiene en cuenta el texto en conjunto, las atracciones a distancia entre fragmentos en algún modo conectados, y finalmente las demás eventuales correcciones intervenidas en estos fragmentos" (Segre 1990, pp. 55-56), pues desde el momento en que los poemas se unen en cadena se ilustran recíprocamente ${ }^{2}$. Por este principio relacionante hay que tener en cuenta que aquellos significados implícitos en un libro de poemas pueden considerarse explícitos para el resto de artes o líricas afines.

Parece conveniente tener unos esquemas aclaratorios de cuáles son los poemas que fueron olvidados ${ }^{3}$, de aquellos otros que fueron cambiados $^{4} y$ de los que incorpora en la edición de 1907 (véase Apéndice I).

2. Como afirmaba Carlos Bousoño (1952, pág. 107), "el concepto de modificante puede tener aún mayor alcance quizá, si pensamos que, dentro de un mismo libro, unos poemas se apoyan en otros y a su lado cobran relieve".

3. Ésta es la serie de poesías olvidadas: 1. Desolaciones y monotonías: La Fuente "Desde la boca de un dragón", Invierno "Hoy la carne aterida", Cenit "Me dijo el agua clara", El Mar Triste "Palpita un mar de acero de olas", Crespúsculo "Caminé hacia la tarde de verano", Otoño "El cárdeno otoño". 2. Del camino: IV "Dime, ilusión alegre", XIV "Siempre que sale el alma de la obscura galería". 3. Salmodias de Abril: Preludio "El pífano de Abril sonó en mi oído", La tarde en el jardín "Era una tarde de un jardín", Nocturno "Sobre el campo de abril", Nevermore "¡Amarga primavera!". 4. Humorismos: La muerte "Aquel juglar burlesco".

4. 1. Desolaciones y monotonías-Soledades: Tarde "Fue clara tarde", Los cantos de los niños "Yo escucho", La noche "Siempre fugitiva y siempre", Horizonte "En una tarde clara". 2. Del camino: 
En la edición de 1907 se observan varios tipos de cambios que modulan nuevamente el texto.

\section{Modificaciones que afectan exclusivamente a la separación estrófica}

La mitad de las correcciones llevada a cabo por Antonio Machado en la edición de Pueyo son correcciones de separación estrófica o paraestrófica ${ }^{5}$ encaminadas a dar una mayor claridad física al poema. Los nuevos versos en blanco que separan una paraestrofa de otra son como las vidrieras por donde entra la claridad al verso haciéndolo más transparente a los sentidos. Ello se debe a que cuando Antonio Machado vuelve a esta poesía de nuevo lo hace desde una óptica más racional, con un espíritu más crítico y estético.

Hay dos criterios básicos de separación estrófica: separación en núcleos semánticos cerrados o separación en núcleos semánticos incompletos. Otros criterios de separación estrófica son los diálogos entre el yo lírico y un interlocutor natural, o incluso las terminaciones tajantes de poemas para los que selecciona dos versos simbólicamente antitéticos que en definitiva resumen el contenido del mismo. En general, el poeta tiende a separar grupos de cuatro versos, quizá porque sean las formas más cercanas al sentir popular, aunque no sea necesariamente pertinente ni por cambio de rima ni por cambio de ritmo estrófico.

\footnotetext{
Preludio "Mientras la sombra pasa de un santo amor", I "Daba el reloj las doce... y eran doce", II "Sobre la tierra amarga", III "En la miseria lenta del camino", V "Crear fiestas de amores", VI "Arde en tus ojos un misterio", VII "iTenue rumor de túnicas que pasan", VIII "iOh, figuras del átrio, más humildes", IX "Quizás la tarde lenta todavía", X "Algunos lienzos del recuerdo tienen", XI "Crece en la plaza en sombra”, XII "Las ascuas de un crepúsculo morado”, XIII "¿Mi amor?... $¿$ Recuerdas, dime", XV "Me dijo un alba de la primavera", XVI " $; O h$, dime, noche amiga, amada vieja".3. Salmodias de Abril-Canciones y Coplas: Canción "Abril florecía", Ocaso "Me dijo una tarde", Campo "La vida hoy tiene ritmo", Mai Piu "Era un mañana y Abril", Fantasía de una noche de Abril "Sevilla?", Tierra Baja "El sueño bajo el sol que aturde", La Mar alegre "El caso roído y verdoso". 4. Humorismos-Humorismos, Fantasías, Apuntes: La noria "La tarde caía", El cadalso "La aurora asomaba", Glosa "Nuestras vidas son los ríos".
}

5. Entiendo por paraestrofas "grupos irregulares de versos cada uno de los cuales no guarda relación formal con los demás --a diferencia de la «estrofa»-, y está motivado en su extensión sólo por el contenido" (Paraíso 1971, pp. 253-54). He aquí la lista de poemas que se ven afectado por cualquiera de las antedichas separaciones: 1. Desolaciones y monotonías: Los cantos de los niños "Yo escucho", Noche "Siempre fugitiva y siempre", Horizonte "En una tarde clara y amplia". 2. Del camino: Preludio "Mientras la sombra pasa", I "Daba el reloj las doce... y eran doce", II "Sobre la tierra amarga", III "En la miseria lenta del camino", V "Crear fiestas de amores", VI "Arde en tus ojos un misterio", VII "iTenue rumor de túnicas que pasan", VIII "¡Oh, figuras del átrio, más humildes", IX "Quizá la tarde lenta todavía", X "Algunos lienzos del recuerdo tienen", XI "Crece en la plaza en sombra", XIII " ¿Mi amor?... ¿Recuerdas, dime", XV "Me dijo un alba de la primavera".3. Salmodias de Abril: Campo "La vida hoy tiene ritmo", Tierra Baja "El sueño bajo el sol que aturde". 4. Humorismos: La noria "La tarde caía", Cadalso "La aurora asomaba", Glosa "Nuestras vidas son los ríos". 
El caso de la silva es particularmente atractivo porque ventisiete de las cuarenta y dos composiciones de las primeras Soledades son silvas, seis de ellas mantienen una rima consonante, diecinueve una rima asonante y dos tienen un tipo de rima libre-alterna. A pesar de ser la silva un poema no estrófico, los poetas suelen dividirlo en formas paraestróficas desiguales (Quilis 1985, pág. 161). No ha de extrañar, por tanto, el distinto número de versos que contengan las estrofas de los poemas compuestos en silvas, pero es anormal tratar de separar un poema compuesto en silvas en estrofas de cuatro versos, máxime cuando, en algunos de los casos, no hay un contenido semántico completo.

En las Soledades de 1903 aparece la "silva arromanzada de endecasílabos y heptasílabos libremente combinados y sin otro enlace que la asonancia uniforme en los versos pares, serie menos corriente en el Modernismo que la de metros fluctuantes de diversas medidas, la cual Machado ensayó una sola vez en la poesía amétrica $O h$, tarde luminosa (Navarro Tomás, 1964, pp. 425-443). En las Soledades de 1907 no sólo tiene el verso como unidad rítmica básica, sino también la estrofa que proporciona una alternancia rítmica visual a base de pareados, tercetos, cuartetos, etc.

\section{Modificaciones que afectan al orden de los poemas}

En cuanto al orden de los poemas que aparecen en la edición de 1903, por lo general se mantiene en la edición de 1907. El proceso de configuración ordinal de este último poemario se basó en una inserción de poemas sobre la edición de 1903. La edición de 1907 se presenta como una edición más abultada respecto a la anterior (Véase Apéndice II).

Los cambios que el autor ha hecho se pueden resumir así: 1. Poemas que aparecen endosados en el nuevo poemario con un nuevo funcionamiento textual ${ }^{6}$. 2. Poemas que no han variado su orden funcional en el poemario ${ }^{7} .3$. Poemas que han sido claramente sustituidos por otros poemas ${ }^{8}$. 4. Poemas que han sido

6. Son los poemas de la sección de Soledades: Tarde, Los cantos de los niños, Noche, Horizonte.

7. Son los siguientes poemas de la sección Del camino: Preludio "Mientras la sombra pasa", I "Daba el reloj las doce", II "Sobre la tierra amarga", III "En la miseria lenta del camino", V "Crear fiestas de amores", VI "Arde en tus ojos un misterio, virgen”, VII "¡Tenue rumor de túnicas que pasan", VIII "¡Oh, figuras del atrio", IX "Quizás la tarde lenta todavía", X "Algunos lienzos del recuerdo tienen", XI "Crece en la plaza en sombra", XII "Las ascuas de un crepúsculo morado", XIII "¿Mi amor?... ¿Recuerdas, dime" y los siguientes de la sección Humorismos: Fantasias, Apuntes, La noria, El Cadalso.

8. En las Soledades están XVIII El poeta "Maldiciendo su destino", XIX " ¡Verdes jardincillos!" que sustituyen a Crepúsculo y Otoño. En la sección Del camino el poema IV "El sol es un globo de fuego" sustituye al IV "Dime, ilusión alegre". 
eliminados sin dejar rastro ${ }^{9} .5$. Poemas que han sido levemente cambiados de orden dentro de la misma sección ${ }^{10}$. 6. Poema que ha sido cambiado de sección ${ }^{11}$.

Es difícil precisar con acierto el porqué de los cambios, tampoco hay que esforzar la actitud crítica para tratar de justificar una serie de modificaciones. Lo más importante es que la base de creación de la edición de Pueyo sigue la línea estructural creada en 1903. El Antonio Machado de 1907 es el que se transparenta en la sección Galerías o Varia y aún ahí el poeta sigue los pasos de su primer poemario.

\section{Transfiguración de la tópica}

Desde 1898, año en el que Antonio Machado empezó a componer poemas, hasta 1907, año de publicación de su segundo libro, han pasado casi 10 años. En los últimos años del siglo XIX y los primeros años del siglo XX se está gestando en España el Modernismo.

La tópica del Modernismo, fuentes, jardines, ocasos, caminos, pueblos abandonados, ríos soñolientos, viejas leyendas, mujeres angelicales, sentimientos de melancolía inundan los primeros poemas de los hermanos Machado, de Villaespesa, Antonio de Zayas, Juan Ramón Jiménez, Rubén Darío. No es el objeto de esta investigación analizar la gestación de esta tópica. Gracias a la precisión semántica y léxica que me han proporcionado distintos grupos de concordancias realizadas sobre las principales obras del Modernismo desde 1900 a 1907, he podido acotar aquella parte de la configuración tópica que cambia desde las Soledades de 1903 a las de 1907. Es decir, son básicos dentro del Modernismo conceptos como lentitud, lejanía, risa, alegría, que Antonio Machado modifica o quita en la edición de 1907. Un análisis pormenorizado de estos conceptos sería interesante porque se podría comprobar la mejora técnica que suponen para la poesía machadiana, pero rebasaría los límites de esta publicación. Lo que propongo aquí es una abstracción sucinta y básica de las principales conclusiones a las que he llegado después del análisis de los cambios de las dos ediciones.

9. En la sección Soledades han sido eliminados: La fuente, Invierno, Cenit, El Mar triste. De la sección Del camino han sido eliminados XVI "Siempre que sale el alma de su oscura galería". De Canciones y Coplas fueron eliminados, Preludio "El Pífano de Abril sonó en mi oído". La tarde en el jardín, Nocturno, Nevermore, y de la sección Humorismos, Fantasías fue eliminada La muerte.

10. El poema que en la sección Del camino de 1903 tenía el número XIV ha desaparecido y este orden lo ocupa en 1907 el poema "Me dijo un alba de la primavera" que en 1903 tenía el número XV. El que en 1903 tenía el número XVI “¡Oh, dime, noche amiga, amada”, pasa en la versión de 1907 a figurar con el número XV. En la sección Coplas y canciones se han cruzado ordinalmente otros dos poemas los que en 1903 llevaban el título Ocaso y Campo y lo mismo ocurre con la pareja de poemas que en 1903 se titulaban La Mar alegre y Tierra baja.

11. Se trata del poema Fantasia de una Noche de Abril "Sevilla?..." que en 1903 estaba Salmodias de Abril mientras que en 1907 aparece en la sección Humorismos, Fantasías, Apuntes. 
Los conceptos que han sido eliminados, sustituidos, o reducido en número en la edición de 1907 y que modulan la tópica del Modernismo machadiano desde su gestación son, como he dicho antes lentitud, lejanía, risa y alegría. Estos cambios manifiestan una evolución de la lírica modernista hacia una búsqueda de una mayor objetividad expresiva y una depuración del simbolismo. Mientras que en 1903 el sentimiento simbolista de lentitud vital del yo lírico impregna desde la analogía conceptos como camino, corazón, rueca, campana y sus equivalentes matices simbólicos, la vida, la intimidad, el tiempo, o la muerte, en los poemas que se mantienen en el edición de 1907, la lentitud es un concepto más objetivo: lento es el viejo mendigo, la hiedra que trepa por la pared y la noria movida por una mula vieja. El Modernismo está dando sus primeros y lentos pasos hacia otra forma de sentir la vida.

La lejanía, el segundo de los conceptos cambiados, ha dado lugar a que se diga que uno de los tópicos del Modernismo es la huida en el tiempo o en el espacio. Huir a países lejanos es un tópico que el Modernismo hereda del Romanticismo: España, Oriente Medio, China, Japón, incluso el África, son extensiones por las que vuelan la fantasía de Víctor Hugo, Byron, los hermanos Schlegel y que después heredaran los modernistas (Foster 1969, pp. 1-22).

\section{Cambios léxicos}

La edición de 1907 incorpora una serie de cambios que afectan al léxico. Cada uno de ellos aporta una mayor precisión al significado poético. Voy a analizar sólo algunos de estos cambios.

En el poema Tarde "Fue una clara tarde, triste y soñolienta", se sustituye "sonó" por "golpeó"; así se acentúa más el sonido producido por el golpe de la puerta de hierro al cerrarse. Unos versos más adelante se sustituye "clara harmonía" por "monotonía" pues es el monótono sonido del agua de la fuente la característica innata de esta fuente machadiana. No se puede decir que el agua de la fuente vierta "clara harmonía", cuando es la propia fuente la que no deja entrever por sus espejos el misterio del pasado. Igualmente es muy acertado el tercer cambio léxico que sufre este poema cuando en los últimos versos se sustituye "triste" por "amarga" pues la monotonía de esta fuente capaz de revivir en la sensibilidad del poeta un estado que pertenece a un recuerdo frustrado lo que le produce más que tristeza es amargura, acritud.

En el poema Los cantos de los niños, el primer cambio es la sustitución de "coplas" por "cantos" y ello creo que fue debido a que en el título figura la palabra cantos y no coplas. En la III parte de este poema se sustituye "A la paz en sombra" por "Jugando, a la sombra", de esta forma el simbolismo interno del poema - la inocencia con la que se nos presenta el misterio de la vida- se agarra más a la analogía que lo genera: unos niños que simplemente juegan cantando en corro viejas canciones. 
Ya dentro de la sección Del camino, en el Preludio hay de nuevo otra sustitución verbal: "leer" reemplaza a "poner". Si tenemos en cuenta que el poema es un ofertorio de sensaciones a la mágica palabra poética, el verbo "poner" se acerca más a "ofrecer" y es por tanto más acertado que la simple lectura de un salmo que se deriva de la edición de 1903.

En el poema III de esta misma sección se sustituye la palabra "borrasca" por "tormenta", términos aparentemente sinónimos. En principio este cambio se nos presenta como el fruto de un mero gusto individual del poeta, pero no es así. "Borrasca" es, al igual que "tormenta", un temporal en el mar con lluvia, viento y relámpagos; en cambio el término "tormenta" tiene una acepción que al simbolismo de esta poesía le viene como anillo al dedo y es que por "tormenta" también entendemos la alteración violenta del estado de ánimo de alguien; además "tormenta" tiene la misma raíz etimológica que "tormento", lo cual acrecienta en mayor medida su valor simbólico etimológico.

En el poema Campo de esta misma sección ha sido sustituida "blanca" por "rápida". Evidentemente en el contexto no importa el color de la corza sino su rapidez, por hacerse inalcanzable para los deseos del yo lírico.

Otro cambio, que a simple vista se nos antoja caprichoso, es el realizado sobre el poema Mai Piu, pero en el fondo demuestra la sutil lectura crítica que realizó Antonio Machado sobre su obra. En este poema se sustituye "alcoba triste" por "triste alcoba". El Oriente en la simbología machadiana y en todo el Modernismo conlleva sentimientos de alegría y dulzura. En la edición de 1903 el adjetivo triste puede ser entendido como un predicativo subjetivo que atribuye al Oriente la cualidad de estar triste. Pero esto no es aceptable desde el punto de vista tópico y por ello en la edición de 1907 antepone "triste" a "alcoba" convirtiéndolo en un adjetivo que no interesa tanto por su función de epíteto sino porque tan sólo puede calificar a "alcoba", dejando el concepto Oriente libre de anfibologías. Otro de los múltiples cambios sufridos por este poema es la sustitución de "plañir" por la palabra "doblar", en dos ocasiones. La expresión "plañir de campanas" es más simbólica en tanto en cuanto se entiende que las campanas tocan a muerto y alguien llora/plañe de dolor. Pero es mucho más acertada la expresión "doblar de campanas" pues se dice que las campanas cuando tocan a muerto doblan.

\section{Cambios Gráficos}

Esta primera edición aparecía al final con una $\mathrm{Fe}$ de erratas en la que la mayoría de ellas son faltas de ortografía que aparecen corregidas, en el caso de que se recoja el poema, en la edición de 1907. Esta edición incorpora también otros cambios gráficos: 1. "Zenit" cambia a "cenit" en el poema IX de Del camino. En el poema Fantasías de una noche de Abril en la sección Salmodias de Abril 
se cambia "dezires", con registro idiomático antiguo por "decires" con grafía modernizada. 2. "áscua" en 1907 pasa a estar correctamente escrito como "ascua" en el poema XII de Del camino" ${ }^{12}$. 2. Frente a las normas que aceptamos actualmente, los monosílabos fué, á, y ó aparecen acentuados en ambas ediciones de principios de siglo. 4. Otra rareza gráfica que aparece en ambas ediciones es la letra mayúscula con la que se escriben tres palabras, Oriente, Primavera y Abril debido a su importancia semántica. 5. Otras dos palabras que deberían haber entrado en la "Fe de erratas" de 1903 son, "canjilón" donde debería leerse "cangilón"13 y donde dice "trajedia" debería poner "tragedia" 14 . Son faltas de ortografía que podrían haber sido cometidas por el propio Antonio Machado. De cualquier forma ningún testimonio nos queda de la época sobre la corrección de pruebas de este libro y es aventurado dar una respuesta sobre quién cometería estos flagrantes errores.

\section{Sustitución de títulos en los poemas y en las secciones}

Si parte de la tópica modernista se difumina tras la sustitución de unos conceptos por otros, otra parte de esa tópica, quizá la más emblemática, se esfuma a través de la supresión de la mayoría de los títulos de los poemas ${ }^{15}$ y de la dedicatoria que algunos de los poemas de 1903 tenían $^{16}$. Debido a que la mayor parte de los poemas que han perdido su título aparecen ahora bajo números romanos $^{17}$, las subdivisiones dentro de los poemas que aparecían en 1903

12. Este es el único cambio sufrido por este poema, con lo que se convierte en el poema menos cambiado de todos. Por otro lado hay bastantes palabras que en la edición de 1903 aparecen o sin acentuar o mal acentuadas. Dichas palabras, en el caso de ser mantenidas en la segunda edición, aparecen corregidas.

13. Esta palabra se encuentra en el verso 5 del poema La Noria.

14. Este error se encuentra en el verso cuatro del poema El Cadalso.

15. Los poemas a los que se les ha mantenido el título son dos, Horizonte y Fantasía de una noche de abril. Sin embargo, nueve poemas han perdido el título, Tarde, Los cantos de los niños, Noche, Canción, Ocaso, Campo, Mai Piu, Tierra Baja, La Mar Alegre y además otros once títulos que no aparecen en la edición de 1907 porque han sido eliminados completamente: La Fuente, Invierno, Cenit, El Mar Triste, Crepúsculo, Otoño, Preludio, Tarde en la Jardín, Nocturno, Nevermore, Muerte.

16. Todas las dedicatorias a sus compañeros en la lucha modernista han desaparecido. Los poetas a los que se les había dedicado algún poema en la edición de 1903 son: A Rubén Darío Los cantos de los niños, la sección Salmodias de Abril a D. Ramón del Valle-Inclán, el Nocturno a Juan Ramón Jiménez, Mai Piu a Francisco Villaespesa y Fantasía de una noche de Abril que está dedicado "Al venerable maestro D. Eduardo Benot". Con la supresión de estas dedicatorias se pierde parte del ambiente modernista en estos poemas. En la edición de 1907 no aparece ningún verso dedicado aunque en el último poema Elogio lleva un recordatorio de Flor de la santidad de Valle-Inclán.

17. Los poemas que tras haber perdido el título aparecen con números romanos son: Soledades: VI «Fue una tarde...», VIII "Yo escucho los cantos...", XVI «Siempre fugitiva y siempre...", XVII "Horizonte, En una tarde clara...". Canciones y coplas: I "Abril florecía...", IV "Me dijo una tarde de la primavera...", V "La vida hoy tiene ritmo..." VI "Era una mañana y Abril sonreía..." VII "E1 caso roido y verdoso", VIII "El sueño bajo el sol...". 
presentadas con esos mismos números, en la edición de 1907 aparecen separadas simplemente por asteriscos ${ }^{18}$. En cambio, hay un poema, el titulado en ambas ediciones La Noria, que mantiene sus números romanos para separar las dos partes de las que consta, porque la sección a la que pertenece, Humorismo, Fantasías, Apuntes, utiliza un título para cada poema y no la habitual numeración romana.

En cuanto al cambio de nombre de las distintas secciones hay que decir que este cambio se hacer por dos motivos: para justificar el título del poemario completo o para suprimir connotaciones tópicas modernistas. En 1903 el libro Soledades se dividía así: Desolaciones y monotonías, Del camino ${ }^{19}$, Salmodias de Abril, Humorismos ("Los grandes inventos"); en cambio en 1907 aparece la siguiente división: Soledades (Del camino, Canciones y coplas, Humorismos, Fantasías, Apuntes), Galerías, Varia. Es decir, se ha sustituido Desolaciones y monotonías por el título genérico del libro que está semánticamente muy cercano al título de esta parte y que por lo tanto, ahora sí, justifica que el libro se llame Soledades. En la edición de 1907 las Soledades contienen las cuatro subsecciones que en 1903 aparecían exentas porque Antonio Machado es consciente de que todo ese material pertenece a su primer libro, a pesar de que aparezca ahora ampliado, y que lo que trae de nuevo la edición de 1907 son las Galerías y Varia, por ello el libro de 1907 se titula Soledades, Galerías. Otros poemas.

La sustitución de la sección Salmodias de Abril por Canciones y Coplas se debe a que se han suprimido el Preludio y el Nocturno, que eran los poemas que junto con Canción y Fantasía de una noche de Abril justificaban el título de esta sección. Pero es que además el poema Fantasía ahora aparece en la siguiente sección y por lo tanto es lógico que el título que le sugiera esta sección sea el de Canciones, pues el poema Canción aparece en primer lugar sin título. La sección Humorismos pasa a denominarse Humorismos, Fantasías, Apuntes. Evidentemente, al haber trasladado el poema de Fantasías de una noche de Abril a esta sección el poeta es consciente de que tiene que variar el título y lo hace dejándose llevar de nuevo por el cambio realizado, de ahí que aparezca Fantasías en este título. Tampoco la sección Galerías es enteramente original dado que en 1903 el poema XIV de la sección Del camino, suprimido en la edición de 1907 , recoge la idea de que el alma tiene galerías de ensueño, matiz simbólico que permanece a lo largo de toda la nueva sección de este poemario ${ }^{20}$.

18. Me refiero a los poemas que en 1903 se titulaban Tarde, Los cantos de los niños, Canción, Mai Piu, Ocaso.

19. Esta sección está compuesta por un Preludio más dieciséis poema numerados con números romanos. Los poemas de todas Ias demás secciones llevan título.

20. En la edición de 1903 la palabra "galería" aparece una sola vez en un poema que en la edición de 1907 es suprimido. En cambio, en esta misma edición aparece una sección entera titulada Galerías, donde se repite este concepto seis veces en seis poemas distintos pero siempre se refiere a las galerías del alma o del recuerdo, como en la edición de 1903. 
Podemos concluir, pues, diendo que, a pesar de que críticos de reconocido prestigio se han negado a admitir una evolución en el autor de las Soledades, a través de este trabajo de investigación se ha intentado demostrar que el primer Antonio Machado fue un lector atento de su obra y el primer crítico de su poesía. Cada cambio realizado sobre la edición de 1903, la separación estrófica, modificaciones léxicas y gráficas, es un paso adelante en el camino hacia la perfección poética, pero es además una transformación consciente de la mentalidad de su autor y por ende de la época en la que empezó a escribir. La depuración del Modernismo se hace a través de la estilización de la tópica, de la supresión de títulos, poemas, y dedicatorias. Por otro lado, se ve claramente que la edición de 1907 fue realizada bajo la pauta estructural y estética de la edición princeps que es, sin duda, uno de los pocos grandes primeros libros.

\section{BIBLIOGRAFÍA}

ALONSO, Dámaso. 1975. "Poesías de las Soledades de 1903, olvidadas por Machado" en Obras completas IV, Madrid, Gredos, pp. 607-628.

BOUSOÑO, Carlos. 1985. Teoría de la expresión poética, Madrid, Gredos, 2 vols.

CERNUDA, Luis. 1957. Estudios sobre poesía española contemporánea, Madrid, Guadarrama.

CRISPO ACOSTA, O. 1929. "Antonio Machado y sus Soledades", Hispania, XII, pp. 225-42.

FOSTER, David William. 1969. "Un índice introductorio de los «tópicos» de la poesía romántica española: Lugares comunes en la lírica de Rivas, Espronceda, Bécquer y Zorrilla", Hispanófila, no 37, pp. 1-22.

GULLÓN, Ricardo. 1960. "Las Soledades de Antonio Machado", Insula, ํำ 158, pp. 1 y 16.

- 1964. Relaciones entre Antonio Machado y Juan Ramón Jiménez, Pisa, Universidad.

MACHADO, Antonio. 1940. Obras (Poesías completas, Juan de Mairena, Sigue hablando Mairena a sus discípulos, Otros sueltos), México, Séneca.

- 1968. Soledades, ed. Rafael FERRERES, Madrid, Taurus.

- 1989. Poesía y prosa, ed. Oreste MACRÍ, Madrid, Espasa-Calpe.

NAVARRO TOMÁS, Tomás. 1964. "La versificación de Antonio Machado", La Torre, XII, nos. 45-46, pp. 425-443.

PARAISO, Isabel. 1971. "El verso libre de Juan Ramón Jiménez en Dios deseado y deseante", Revista de Filología Española, LIV, pp. 252-269.

QUILIS, Antonio. 1985. Métrica española, Barcelona, Ariel. 
RIBBANS, Geoffrey. 1962. “Antonio Machado's Soledades (1903); A critical Study”, Hispanic Review, XXX, pp. 194-215.

ROMERO LÓPEZ, Dolores. 1993. Concordancia y variantes de las Soledades de Antonio Machado, Salamanca, Universidad.

SEGRE, Cesare. 1990. Semiótica filológica (Textos y modelos culturales), Murcia, Universidad.

\section{APÉNDICE I}

Ésta es la lista de los nuevos que se incorporan en la edición de 1907. El asterisco $\left({ }^{*}\right)$ significa que en ese lugar aparece un poema tomado de la edición de 1903. 1. Soledades: I El viajero "Está en la sala familiar", II "He andado muchos caminos", III "La plaza y los naranjos encendidos", IV En el entierro de un amigo "Tierra le dieron una tarde horrible", V Recuerdo Infantil "Una tarde parda y fría", *, VII "El limonero lánguido suspende", *, IX Orillas del Duero "Se ha asomado una cigueña", X "A la desierta plaza", XI "Yo voy soñando caminos", XII "Amada, el aura dice", XIII "Hacia un ocaso radiante", XIV Cante Hondo "Yo meditaba absorto, devanando", XV "La calle en sombra", *, *, XVIII El poeta "Maldiciendo su destino", XIX "iVerdes jardincillos!”. 1.2. Del camino: *,*, *, IV "El sol es un globo de fuego", *, *, *, *, *, *, *, *, *, *, XVI "Al borde de un sendero nos sentamos", XVII "Es una forma juvenil que un día". 1.3. Canciones y coplas: *, II De la vida, Coplas, Elegiacas, "iAy del que llega”, III Inventario galante "Tus ojos me recuerdan”, *, *, *, *, * 1.4. Humorismos, fantasías, apuntes: *, *, Las moscas "Vosotras, las familiares", Elegia de un madrigal "Recuerdo que una tarde", Acaso "Como atento no más a mi quimera", Jardín "Lejos de tu jardín quiera la tarde," *, A un naranjo y un limonero visto en una tienda de plantas y flores, Los sueños malos, Hastío "Pasan las horas de hastío", Consejos "Este amor que quiere ser", *. 2. Galerías: Introducción "Leyendo, un claro día", I "Desgarrada la nube, el arco iris", II "Y era el demonio de mi sueño el ángel", III "Desde el umbral de un sueño me llamaron", VI Sueño Infantil: "Una clara noche", V "Si yo fuera un poeta", VI "Llamó a mi corazón", VII "Hoy buscarás en vano", VIII "Y nada importa ya que el vino de oro", IX "Tocados de otros días", X "La casa tan querida", XI "Ante el pálido lienzo", XII "Tarde tranquila", XIII "Yo, como Anacreonte", XVI "Y no es verdad, dolor, yo te conozco", XVII "Y ha de morir contigo el mundo mago", XVIII "Desnuda está la tierra", XIC Campo "La tarde está muriendo", XX A un viejo distinguido señor "Te he visto, parque", XXI Los sueños "El hada más hermosa ha sonreído", XXII "Guitarra del mesón que hoy sueñas jota", XXIII "El rojo sol de un seuño en el Oriente asoma", XXIV "La primavera besaba", XXV Renacimiento "Galerías del alma...", XXVI "En nuestras almas, todo", XXVII "Tal vez la mano, en sueños", XXVIII "Y podrás conocerte recordando", XXIX "Los árboles conservan", XXX "Húmedo está, bajo el laurel, el bando", 3. Varia: Caballitos "Pegasos, lindos pegasos", Ruidos "Deletreos de armonía", Pesadilla "En medio de la plaza", De la vida: "Poeta ayer, hoy triste y pobre", Sol de invierno "Es medio día. Un parque", Elogios "Esta leyenda en sabio romance campesino". 


\section{APÉNDICE II}

Veamos primero un esquema básico para comprobar como se organiza el nuevo texto: (*** significa poema de 1903 insertado en la edición de Pueyo)

\section{Desolaciones y monotonías-Soledades}

$\begin{array}{ll} & \text { I El viajero: "Está en la sala familiar" } \\ \text { II "He andado muchos caminos" } & \\ \text { III "La plaza y los naranjos encendidos" } & \text { IV En el entierro de un amigo "Tierra" } \\ & \text { V Recuerdo Infantil "Una tarde parda y fría" } \\ & * * * \text { Tarde "Fue una tarde triste" } \\ & \text { VII "El limonero lánguido suspende" } \\ & \text { *** "Yo escucho los cantos" } \\ \text { La Fuente } & \text { IX Orillas del Duero "Se ha asomado" } \\ \text { Invierno } & \text { X "A la desierta plaza" } \\ \text { Cenit } & \text { XI "Yo voy soñando caminos" } \\ \text { Mar Triste } & \text { XII "Amada, el aura dice" } \\ & \text { XIII "Hacia un ocaso radiante" } \\ & \text { XIV Cante Hondo "Yo meditaba absorto," } \\ & \text { XV "La calle en sombra" } \\ & \text { *** Noche "Siempre fugitiva y siempre" } \\ & \text { *** Horizonte "En una tarde clara" } \\ \text { Crepúsculo } & \text { XVIII El poeta "Maldiciendo su destino" } \\ \text { Otoño } & \text { XIX "iVerdes jardincillos" }\end{array}$

\section{Del camino}

*** Preludio: "Mientras la sombra pasa" *** I "Daba el reloj las doce" *** II "Sobre la tierra amarga" *** III "En la miseria lenta del camino"

IV. "Dime..."IV "El sol es un globo de fuego"

*** V "Crear fiestas de amores"

*** VI "Arde en tus ojos un misterio, virgen"

*** VII " ¡Tenue rumor de túnicas que pasan"

*** VIII " $\mathrm{OOh}$, figuras del atrio,"

*** IX "Quizás la tarde lenta todavía"

*** X "Algunos lienzos del recuerdo tienen"

*** XI "Crece en la plaza en sombra"

*** XII "Las ascuas de un crepúsculo morado" 
XVI "Al borde de un sendero"

XVII "Es una forma juvenil que un día"

3. Salmodias de Abril. Canciones y coplas

Preludio

*** Canción "Abril florecía"

La tarde II De la vida, coplas elegiacas

III Inventario galante "Tus ojos"

Ocaso *** IV "Me dijo una tarde"

Campo *** V "La vida hoy tiene ritmo"

Nocturno *** VI "Era una mañana y Abril sonreía"

Fantasía *** VII "El casco roído y verdoso"

Nevermore *** VIII "El sueño bajo el sol que aturde"

\section{Humorismos. Humorismos, Fantasías, Apuntes}

*** La Noria "La tarde caía"

*** El Cadalso "La aurora asomaba"

La Muerte . Las moscas: "Vosotras, las familiares"

Elegía de un madrigal "Recuerdo..."

Acaso... "Como atento no más"

Jardín: "Lejos de tu jardín quiera la tarde"

Fantasía de una noche de Abril "Sevilla?... Granada?..."

A un naranjo y un limonero...

Los sueños malos

Hastio: "Pasan las horas de hastío"

Consejos "Este amor que quiere ser"

*** Glosa "Nuestras vidas son los ríos" 